\title{
PATHOPHYSIOLOGY OF WDR45 MUTATION SENDA / BPAN IN AUTOPHAGY DISEASE
}

\author{
K.Tsukida1), K. Muramatsu1), H. Shimizu²), H. Osaka1), T. Yamagata1). \\ 1) Jichi Medical University, Pediatrics, Shimotsuke, Japan. \\ 2) Brain Research Institute Niigata University, Pathology, Niigata, Japan.
}

\section{Background}

It is well known that autophagy is an essential biological mechanism for all organisms. Static encephalopathy of childhood with neurodegeneration in adulthood (SENDA) or beta propeller protein associated neurodegeneration (BPAN) is a disease caused by autophagy dysfunction. SENDA / BPAN is based on WDR45 mutation at Xp11.23 and is a part of the novel neurodegeneration with brain iron accumulation (NBIA). Its pathophysiology is largely unknown.

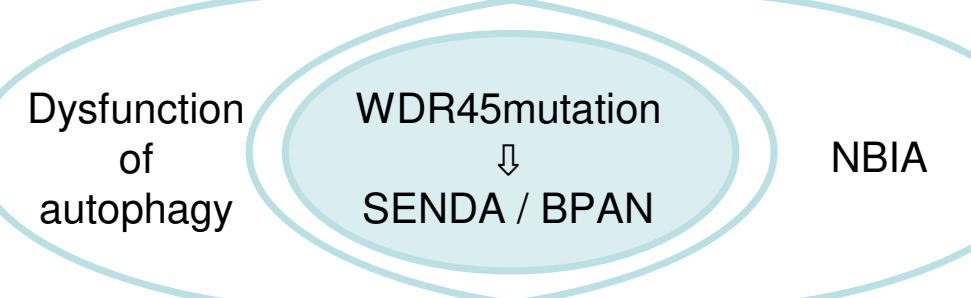

Fig1. Disease classification of SENDA / BPAN

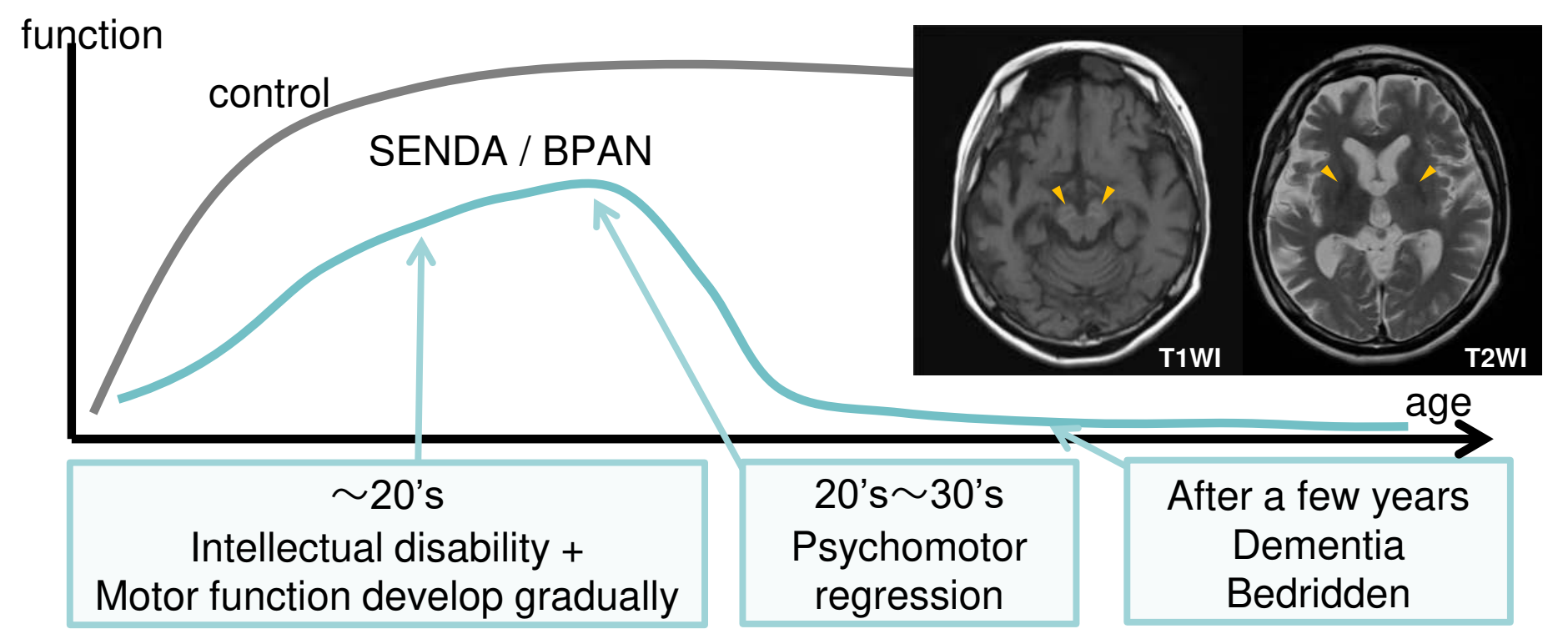

Fig2. Clinical course of SENDA / BPAN

\section{Method}

WIPI-4, LC3 the maker of autophagosome, and p62 the substrate of autophagy expression was analyzed by immunohistochemistry and qRT-PCR using RNA extracted from the substantia nigra (SN), globus pallidus (GP), thalamus (Th), frontal cortex (FC), and cerebellar cortex (CC) of post-mortem human brains obtained from SENDA/BPAN patient and three control patients.

\section{Results}

\begin{tabular}{|c|c|c|c|c|c|}
\hline Case & Mutation & Initial walking & Speech ability & Epilepsy & Psychomotor regression \\
\hline 1 & c. $400 \mathrm{C}>\mathrm{T}($ p.Arg134*) & NA & No word & + & Static \\
\hline 2 & c. $400 \mathrm{C}>\mathrm{T}($ p.Arg134*) & NA & No word & + & Static \\
\hline 3 & c. $831-1 G>C$ & NA & No word & + & Progressive \\
\hline 4 & c.868C>T:p.Gln290* & NA & No word & + & Progressive \\
\hline 5 & c. $830+1 G>A$ & $1 \mathrm{y} 7 \mathrm{~m}$ & No word & + & $\begin{array}{l}\text { Progression from } 1 \text { to } \\
\text { 4years, and stable }\end{array}$ \\
\hline 6 & $\begin{array}{c}\text { c.516G>C } \\
\text { (p.Asp174Valfs*29) }\end{array}$ & $2 y 7 m$ & One word & + & $\begin{array}{l}\text { Progressive from } \\
\text { teenage }\end{array}$ \\
\hline 7 & c.519+1_519+3del & 1 y $6 m$ & One word & + & Progressive \\
\hline 8 & $\begin{array}{c}\text { c.439+1G>T (p.[Gly147Val; } \\
\text { Val147_Leu148ins8) }\end{array}$ & $3 y$ & No word & + & Progressive \\
\hline 9 & $\begin{array}{c}\text { c.1033_1034dup } \\
\left.\text { (p.Asn345Lysfs }{ }^{*} 67\right)\end{array}$ & 1 y $6 m$ & Few words & + & Progressive \\
\hline 10 & c.322del (p.Ser108Leufs*10) & 1 y $3 m$ & No word & + & Progressive from 28years \\
\hline 11 & c.437dup ( p.Leu148Alafs ${ }^{\star} 3$ ) & $2 y 2 m$ & No word & + & Progressive \\
\hline 12 & c. $19 \mathrm{C}>\mathrm{T}\left(\mathrm{p} . \operatorname{Arg} 7^{\star}\right)$ & $1 \mathrm{y} 6 \mathrm{~m}$ & Three words & + & Progressive from 30years \\
\hline 13 & c. $830+2 \mathrm{~T}>\mathrm{C}$ & $3 y$ & One word & + & Progressive from 21 years \\
\hline 14 & c.637C>T (p.Gln213*) & $1 \mathrm{y} 6 \mathrm{~m}$ & Two-word sentences & - & Progressive \\
\hline 15 & c. $56-2 A>G$ & $2 \mathrm{y} 0 \mathrm{~m}$ & Two-word sentences & + & Progressive from 38years \\
\hline 16 & c. $729-2 A>G$ & NA & No word & + & Static \\
\hline
\end{tabular}

Table. Clinical characteristics

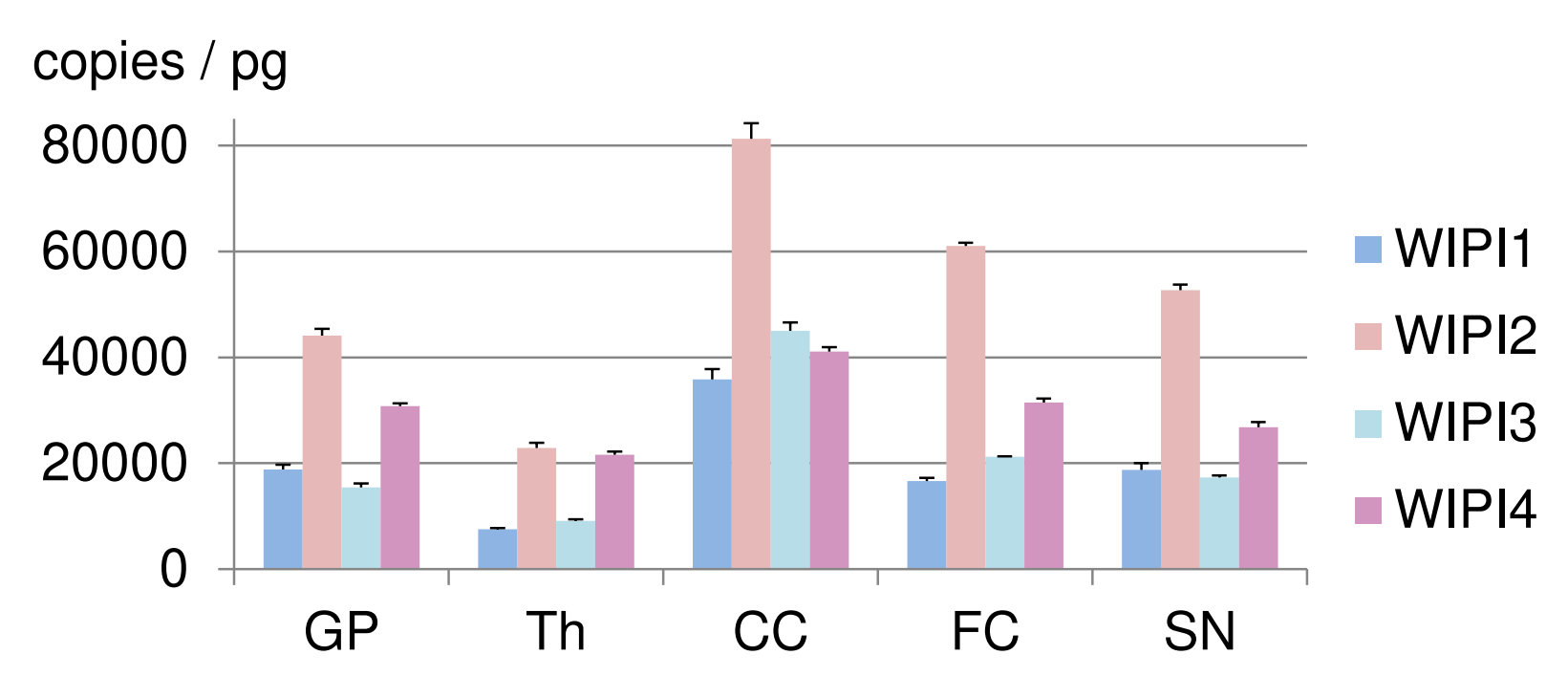

Fig3. WIPIs expression of SENDA / BPAN patient

WDR45 encodes the WIPI-4 protein which has three additional isoforms, WIPI-1, 2, and 3. WIPI-2 was the most dominant isoform, followed by WIPI-4 in most regions of SENDA/BPAN patient. Same tendencies were also indicated in the three control patients (data not shown).

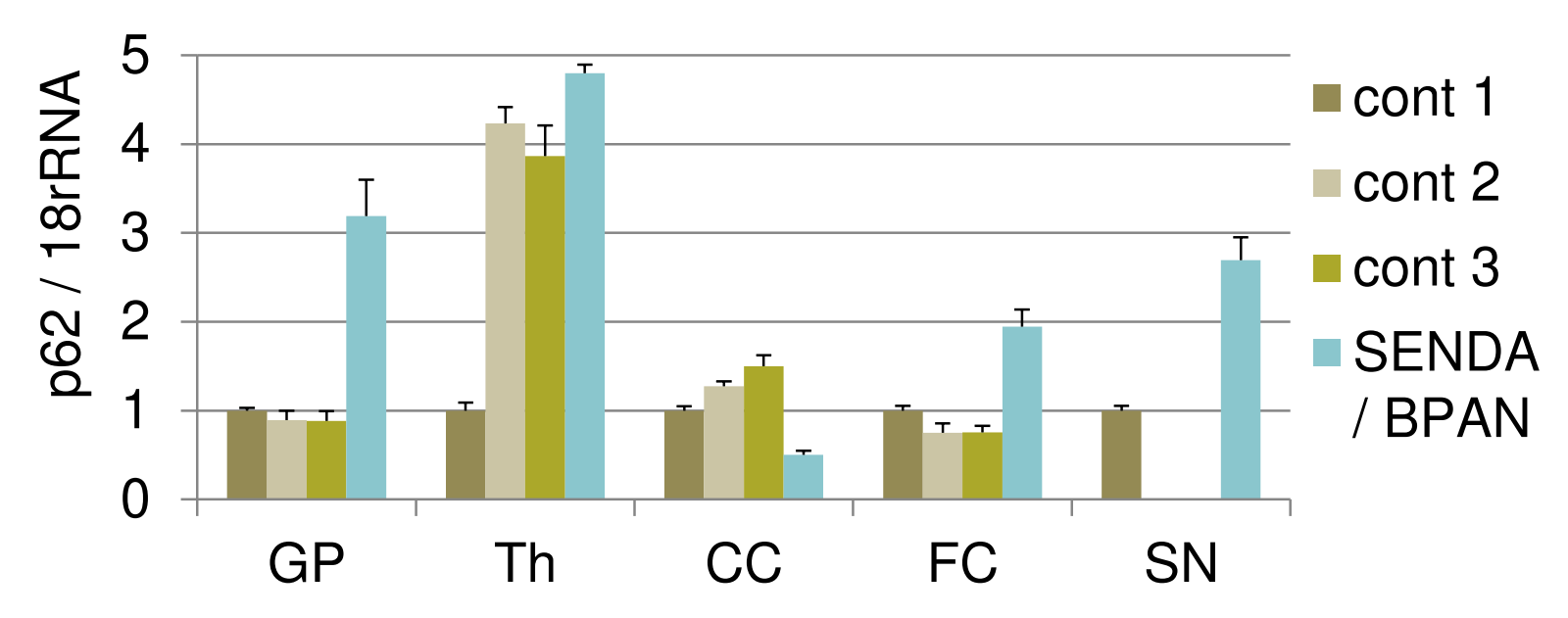

Fig4. p62 expression of SENDA / BPAN and the three control patients

The p62 expression was high in the SN, GP and FC of SENDA patient compared to control patients. On the other hand, p62 expression was low in the CC of SENDA / BPAN patient compared to other control patients.

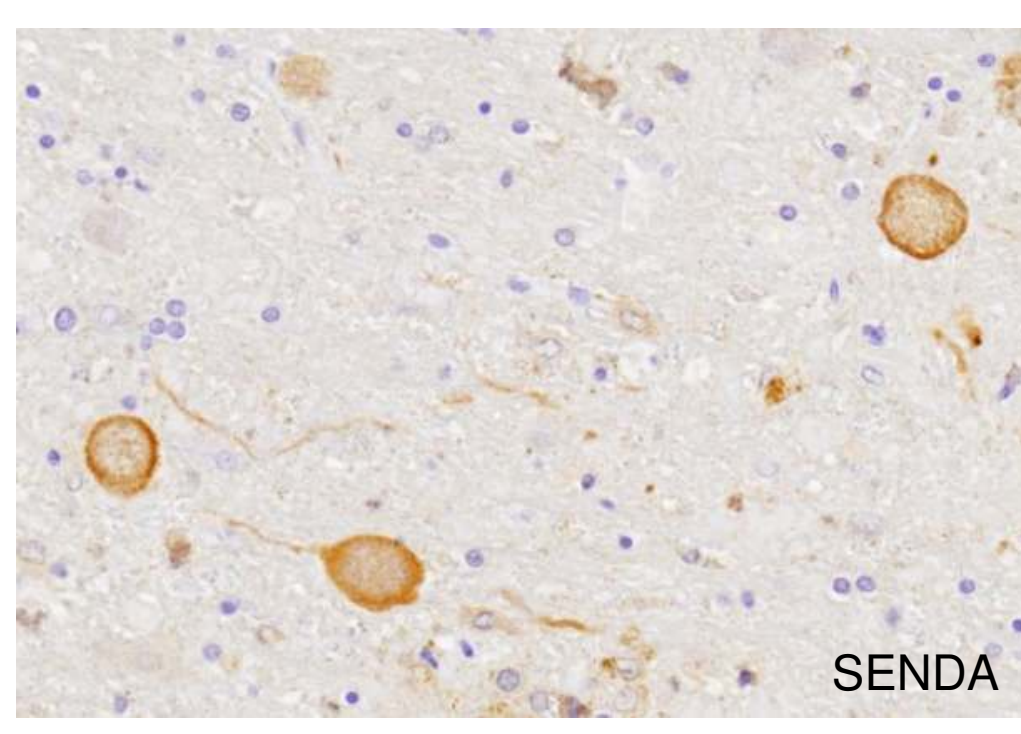

Fig5. Pathological features of the GP

LC3 was stained more strongly and neurospheroids were remarkable in the GP of SENDA patient. On the other hand, no LC3 staining and neurospheroids were seen in the GP of control patient.

(×100, LC3 immunohistochemistry)

\section{Discussion}

- Why are severity and genotype not correlated with?

WDR45 is located on the $\mathrm{X}$ chromosome, hence subjects with $\mathrm{X}$ inactivation are predominantly affected.

- Why are MRI findings remarkable in the SN and GP ?

Although WIPI-4 expression is not vary in SENDA / BPAN and control patients, p62 is enhanced in the SN and the GP. Additionally, LC3 staining is enhanced in the GP. Under these conditions, autophagy must be impaired in the SN and GP and it leads to MRI findings .

\section{Conclusion}

The reason why the $\mathrm{SN}$ and GP experience severe accumulation of iron remains unclear because WIPI-4 is dominant in all regions. However, an increase of p62 expression and LC3 staining suggests an insufficiency of autophagy in the SN and GP.

\author{
Reference \\ 1. Saitsu H, et al., Nat Genet. 2013; 45: 445-9. \\ 2. Muramatsu K. No To Hattatsu. 2016; 48: 177-83. \\ 3. Ebrahimi-Fakhari D, et al., Brain. 2016; 139: 317-37.
}

